\title{
Types of Derivatives: Concepts and Applications (II)
}

\author{
Salma A. Khali1 ${ }^{1}$, Mohammed A. Basheer ${ }^{2}$ \& Tarig A. Abdelhaleem ${ }^{3}$ \\ ${ }^{1}$ Department of Mathematics, Faculty of sciences, Princess Nourah bint Abdulrahman University, Riyadh, KSA \\ ${ }^{2}$ Department of Mathematics, Faculty of sciences, University of Alnillin, Khartoum, Sudan \\ ${ }^{3}$ Department of Mathematics, Collage of Applied and Industrial sciences, University of Bahri, Khartoum, Sudan \\ Correspondence: Salma A. Khalil, Hepatobiliary Sciences \&Liver transplant- 144National Guards Hospita Po box 22490 \\ Riyadh 11426, KSA. E-mail: Salma-math@ windowslive.com
}

Received: October 12, $2016 \quad$ Accepted: December, $82016 \quad$ Online Published: January 23, 2017
$\begin{aligned} & \text { doi:10.5539/jmr.v9n1p50 } \\ & \text { URL: http://dx.doi.org/10.5539/jmr.v9n1p50 }\end{aligned}$

\begin{abstract}
The notion of differential geometry is known to have played a fundamental role in unifying aspects of the physics of particles and fields, and have completely transformed the study of classical mechanics.

In this paper we applied the definitions and concepts which we defined and derived in part (I) of our paper: Types of Derivatives: Concepts and Applications to problems arising in Geometry and Fluid Mechanics using exterior calculus. We analyzed this problem, using the geometrical formulation which is global and free of coordinates.
\end{abstract}

Keywords: differential geometry, exterior calculus, free of coordinates

\section{Introduction}

In our previous paper, we defined three types of major derivatives such as the Exterior Derivative, Lie Derivative and Covariant Derivative (Kolár, et al., 1999; Warner, 2013). We divided our work into two parts, where in the first section we started by defining a Differentiable Manifold structure, (Arkani-Hamed, et al., 2010) then The Tangent Bundle, where we built the Bundle from the tangent space defined on a Differentiable Manifold; we defined the Cotangent Bundle in a similar fashion to the Tangent Bundle, then considered Smooth Vector Fields and finally concluded our structure by defining Tensor fields and Riemannian Manifolds (Beig, R.).

In the second part, we defined The Covariant Derivative in which we defined Covariant derivatives of covectors and Tensors, Lie Derivatives:Lie Derivatives of tensor fields and Differential Forms. Finally, in the third part we defined The Exterior Derivative and shed light on the properties of the respective Derivative.

Exterior calculus is a concise formalism to express differential and integral equation on smooth and curved spaces in a consistent manner, while revealing the geometrical invariants at play. One of the main goals of developing a geometric theory of fluid is to put all the existing computational techniques in one abstract setting. This rationalization of computational mechanics will be theoretically interesting for its own sake.

Rewriting equations of fluid mechanics in terms of differential forms enables one to clearly see the geometric features of the fluid field theory.

\section{Main Formulas}

2.1 Definition (Kobayashi, S., \& Nomizu, K., 1963)

Let $\mathrm{M}$ be a smooth differentiable manifold of dimension $\mathrm{m}$. The Tangent Bundle TM is defined to be

$$
\mathrm{TM}=\mathrm{U}_{\mathrm{p} \in \mathrm{M}} \mathrm{T}_{\mathrm{p}} \mathrm{M}=\left\{(\mathrm{p}, \mathrm{v}) \mid \mathrm{p} \in \mathrm{M}, \mathrm{v} \in \mathrm{T}_{\mathrm{p}} \mathrm{M}\right\} .
$$

2.2 Definition (Lang, S., 1999)

AVector field $v$ on $M$ is a section of the tangent bundle TM, ie $v: M \rightarrow T M$ such that $\pi \circ v(p)$ for every $p \in M$. In other words Let $x: U \rightarrow R^{m}$ be a local chart of $M$, and $p \in U$, then $v(p)=\sum_{i=1}^{m} v_{p}\left(x^{i}\right) \frac{\partial}{\partial x^{i}} l_{p}$. avector field on $M$ is a map $v$ which assigns to each point $p \in M$ a tangent vector $v(p)=v_{p} \in T_{p}(M)$. 


$$
g_{U V}(p)=\left.\left(\frac{\partial y^{i}}{\partial x^{j}}\right)_{1 \leq i, j \leq m}\right|_{\phi_{U}(p)} .
$$

\subsection{Definition}

A covariant derivative is an operator $\mathrm{V}$ on tensor fields which satisfies the following conditions:

1) If $\mathrm{T}$ is of rank $(\mathrm{r}, \mathrm{s})$, then $\nabla \mathrm{T}$ is of rank $(\mathrm{r}, \mathrm{s}+1)$; the covariant rank increases by 1 .

2) For any function $f, \nabla(f)=d f$. $\left[\nabla_{a}(f)=\partial_{a}(f)\right]$

3) For any function $f$ and tensor $T, \nabla(f T)=d f \otimes T+f \nabla T .\left[\nabla_{a}\left(f T_{\ldots}^{\cdots}\right)=\partial_{a}(f) T_{\ldots}^{\ldots}+f \nabla_{a} T_{\ldots} \ldots\right]$.

4) More generally, for any tensors Sand $\mathrm{T}, \nabla(\mathrm{S} \otimes \mathrm{T})=\nabla \mathrm{S} \otimes \mathrm{T}+\mathrm{S} \otimes \nabla \mathrm{T}$.

2.4 Definition Lie Derivative (O'neill, B., 1983)

If $\varphi$ is a local diffeomorphism $\mathrm{M} \rightarrow \mathrm{N}$, we may define a pull- back map

$\varphi^{*}: \Gamma \mathrm{T}_{\mathrm{r}, \mathrm{s}} \mathrm{N} \rightarrow \Gamma \mathrm{T}_{\mathrm{r}, \mathrm{s}}$ Mon mixed field as follows. For $\mathrm{T} \in \Gamma \mathrm{T}_{\mathrm{r}, \mathrm{s}} \mathrm{N}$ we define

$$
\varphi^{*}(\mathrm{~T})_{\mathrm{m}}:=\left(\mathrm{d}_{\mathrm{m}} \varphi\right)^{*} \mathrm{~T}_{\varphi(\mathrm{m})} .
$$

\subsection{Definition}

Let $X \in \mathfrak{X}(\mathrm{M})$ be smooth vector field. Then for every $\mathrm{m} \in$ Mwe denote by $\mathrm{t} \mapsto \varphi_{\mathrm{X}}^{\mathrm{t}}(\mathrm{m})$ the (maximal) integral curve for $X$ with initial point $\mathrm{m}$. the domain of this integral curve is an open interval $\mathrm{I}_{\mathrm{X}, \mathrm{m}}$ containing 0 . Let $\mathrm{T} \in$ $\Gamma \mathrm{T}_{\mathrm{r}, \mathrm{s}} \mathrm{M}$ and we define the Lie derivative of $\mathrm{T}$ with respect to $\mathrm{X}$ by

$$
(\mathcal{L} \times \mathrm{T})_{\mathrm{m}}:=\left.\frac{\mathrm{d}}{\mathrm{dt}}\right|_{\mathrm{t}=0}\left[\left(\varphi_{\mathrm{X}}^{\mathrm{t}}\right)^{*} \mathrm{~T}\right]_{\mathrm{m}}
$$

note that $\varphi_{\mathrm{X}}^{\mathrm{t}}$ is a diffeomorphism from a neighborhood of $\mathrm{m}$ onto a neighborhood of $\varphi_{\mathrm{X}}^{\mathrm{t}}(\mathrm{m})$. Accordingly, the expression

$$
\left[\left(\varphi_{\mathrm{X}}^{\mathrm{t}}\right)^{*} \mathrm{~T}\right]_{\mathrm{m}}
$$

is a well-defined element of $\left(\mathrm{T}_{\mathrm{m}} \mathrm{M}\right)_{\mathrm{r}, \mathrm{s}}$ which depends smoothly on $\mathrm{t}$ (in a neighborhood of 0$)$. Accordingly, $(\mathcal{L} \times \mathrm{T})_{\mathrm{m}}$ defines a tensor in $\left(\mathrm{T}_{\mathrm{m}} \mathrm{M}\right)_{\mathrm{r}, \mathrm{s}}$. Moreover, by the smoothness of the flow of the vector field $\mathrm{X}$ it follows that the section $\mathcal{L} \times \mathrm{T}$ of the tensor bundle $\mathrm{T}_{\mathrm{r}, \mathrm{s}} \mathrm{M}$ thus defined is smooth. In other words, we have defined a linear map.

$$
\mathcal{L}_{\mathrm{X}}: \Gamma \mathrm{T}_{\mathrm{r}, \mathrm{s}} \mathrm{M} \rightarrow \Gamma \mathrm{T}_{\mathrm{r}, \mathrm{s}} \mathrm{M},
$$

called The Lie derivative. In a similar way it is seen that the Lie derivative defines a linear map $\mathcal{L}_{\mathrm{X}}: \mathrm{E}_{\mathrm{k}}(\mathrm{M}) \rightarrow \mathrm{E}_{\mathrm{k}}(\mathrm{M})$ (Cartan's formula) let X be a smooth vector field on M. then on E(M),

$$
\mathcal{L}_{\mathrm{X}}=\mathrm{i}(\mathrm{X}) \text { o d }+\mathrm{d} \text { o } \mathrm{i}(\mathrm{X}) \text {. }
$$

The proof was given in paper (I)Applications.

\section{The Geometric Setup}

We give a geometric model of the basic kinematics used in modeling the fluid flow.

\subsection{The Fluid Space (Haller, G., 2001)}

Assume our fluid flows in a smooth manifold $\mathrm{M}(\mathrm{M}$ denotes a differentiable n-manifold).

A fluid particle is a point in the manifold. Points in a domain $\mathrm{D} \subset \mathrm{M}$ represent the geometric positions of material particles;these points are denoted by $\mathrm{x} \in \mathrm{M}$ and called particle labels.

\subsection{Fluid Motion (Geometric Notion of the Fluid Motion)}

The Fluid moves in a manifold whose points represent the fluid particles. Let $x \in M$ be a point in $M$ ( $M$ is the space in which the fluid moves) and consider the particle of fluid moving through $\mathrm{x}$ at time $\mathrm{t}=0$. As $\mathrm{t}$ increases, we denote by $\phi_{\mathrm{t}}(\mathrm{x})$ the curve followed by the fluid particle, which is initially at $\mathrm{x} \in \mathrm{M}$. For fixed $\mathrm{t}$, each $\phi_{\mathrm{t}}$ will be a diffeomorphism of M. Thus the fluid motion is a smooth one parameter family of diffeomorphisms $\phi_{\mathrm{t}}: \mathrm{M} \rightarrow \mathrm{M}$; with $\phi_{0}=\mathrm{Id}$.

$t \rightarrow \phi(t)$ is a one parameter family of diffeomorophisms of M.For each value oft,we define a vector field $X_{t} \in \mathfrak{x}(M)$ as follows:

For $\mathrm{x} \in \mathrm{M}, \mathrm{X}_{\mathrm{t}}(\mathrm{x})$ is the tangent vector to the curve $\mathrm{u} \rightarrow \phi(\mathrm{u}) \phi(\mathrm{t})^{-1} \mathrm{x}$ at

$\mathrm{u}=\mathrm{t}, \mathrm{X}$, is the velocity field corresponding to the fluid motion defined by $\phi(t)$ : that is, $\mathrm{X}_{\mathrm{t}}(\mathrm{x})$ is the velocity vector of the particle that, at time $\mathrm{t}$, is at the point $\mathrm{x}$. 


$$
\begin{gathered}
X\left(\phi_{\mathrm{t}}(\mathrm{x}), \mathrm{t}\right):=\frac{\partial \phi_{\mathrm{t}}}{\partial \mathrm{t}}(\mathrm{x}) . \\
\mathrm{X}_{\mathrm{t}}(\mathrm{f})(\mathrm{X})=\left.\frac{\mathrm{d}}{\mathrm{du}} \mathrm{f}\left(\phi_{\mathrm{u}} \phi_{\mathrm{t}}^{-1}(\mathrm{x})\right)\right|_{\mathrm{u}=\mathrm{t}}=\left.\frac{\mathrm{d}}{\mathrm{du}}\left(\phi_{\mathrm{u}} \phi_{\mathrm{t}}^{-1}\right)(\mathrm{f})(\mathrm{x})\right|_{\mathrm{u}=\mathrm{t}} \\
=\left.\frac{\mathrm{d}}{\mathrm{du}}\left(\left(\phi_{\mathrm{t}}^{-1}\right)^{*} \phi_{\mathrm{u}}^{*}\right)(\mathrm{f})(\mathrm{x})\right|_{\mathrm{u}=\mathrm{t}}=\left(\phi_{\mathrm{t}}^{-1}\right)^{*}\left(\left.\frac{\mathrm{d}}{\mathrm{du}} \phi_{\mathrm{u}}^{*}(\mathrm{f})(\mathrm{x})\right|_{\mathrm{u}=\mathrm{t}}\right. \\
\text { Or }\left.\phi_{\mathrm{t}}^{*}\left(\mathrm{X}_{\mathrm{t}}(\mathrm{f})\right)(\mathrm{x})\right|_{\mathrm{u}=\mathrm{t}}=\frac{\mathrm{d}}{\mathrm{du}} \phi_{\mathrm{u}}^{*}(\mathrm{f})(\mathrm{x})=\frac{\partial}{\partial \mathrm{t}} \phi_{\mathrm{t}}^{*}(\mathrm{f})(\mathrm{x}) \\
\text { Therefore } \mathrm{X}_{\mathrm{t}}(\mathrm{f})=\left(\phi_{\mathrm{t}}^{-1}\right)^{*} \frac{\partial}{\partial \mathrm{t}} \phi_{\mathrm{t}}^{*}(\mathrm{f}) ; \text { For } \mathrm{f} \in \mathrm{F}(\mathrm{M})
\end{gathered}
$$

Note: the inverse maps be computed by reversing time, $\phi_{\mathrm{t}}^{-1}=\phi_{-\mathrm{t}}$.

A flow is called steady (or stationary)if its vector field satisfies:

$$
\frac{\partial \mathrm{X}}{\partial \mathrm{t}}=0,
$$

i.e. the "shape" of the fluid flow is not changing. Even if each particle is moving under the flow, the global configuration of the fluid does not change.

Let the one-form $\alpha \in \Omega^{1}(\mathrm{M})$ describe the velocity of a fluid. We define vorticity as

$$
\varpi=\mathrm{d} \alpha
$$

The vorticity is a 2 -form UJ is dual to the vorticity vector field (.The trajectories of vorticity field are called vortex lines.A flow is called irrotational if

$$
\varpi=0
$$

From the Poincare lemmaon some open subset $\mathrm{U} \subset \mathrm{M}$, there exist $\varphi \in \mathcal{F}_{\mathrm{U}}$ such that

$$
\alpha=\mathrm{d} \varphi
$$

$\varphi \in \mathcal{F}_{\mathrm{U}}$ is called velocity potential.

\section{Continuity Equation (Erbar, M., 2010; Bajura, R., \& Jones, E., 1976)}

\subsection{Continuity Equation on Manifolds}

Reformulating the continuity equation from the point of view of vector fields and differential forms on manifolds.

Let $\mathrm{D} \subset \mathrm{M}$ be a sub region of MConsider a fluid moving in a domain $\mathrm{D} \subset \mathrm{M}$ and suppose $\omega$ is a fixed $\neg$ volume element differential form on $\mathrm{M}$, which is point-wise nonvanishing. Then, the total mass of the fluid in the region $\mathrm{D}$ at time $\mathrm{t}$ is

$$
m(D, t)=\int_{D} \rho_{t} \omega .
$$

Where $\rho_{\mathrm{t}}$ describes the mass-density of the fluid at time t. from the principle of mass conservation in fluid dynamics, the total mass of the fluid, which at time $\mathrm{t}=0$ occupied a region $\mathrm{D}$,remains unchanged after time $\mathrm{t}$.

Thus, the total mass of the fluid at time $t=0$ occupinga region $D$ is maintained with time, i.e.

$$
\int_{\phi_{\mathrm{t}}(\mathrm{D})} \rho_{\mathrm{t}} \omega=\int_{\mathrm{D}} \rho_{0} \omega \text {. }
$$

Where $\phi_{\mathrm{t}}$ the one-parameter family of diffeomorophisms.Equation (12) isis the integral invariant for conservation of mass. By the change of variable formula, the left hand side of this relation is equal to

$$
\int_{\mathrm{D}} \phi_{\mathrm{t}}^{*}\left(\rho_{\mathrm{t}} \omega\right)=\int_{\mathrm{D}} \rho_{0} \omega .
$$

differentiating,with respect to $t$, we get

$$
\frac{\partial}{\partial \mathrm{t}} \int_{\mathrm{D}} \phi_{\mathrm{t}}^{*}\left(\rho_{\mathrm{t}} \omega\right)=\int_{\mathrm{D}} \frac{\partial}{\partial \mathrm{t}}\left(\phi_{\mathrm{t}}^{*}\left(\rho_{\mathrm{t}} \omega\right)\right)=0
$$

Since $\frac{\partial}{\partial \mathrm{t}}\left(\phi_{\mathrm{t}}^{*}\left(\rho_{\mathrm{t}} \omega\right)\right)=\phi_{\mathrm{t}}^{*}\left(\mathrm{~L}_{\mathrm{X}_{\mathrm{t}}}\left(\rho_{\mathrm{t}} \omega\right)\right)+\phi_{\mathrm{t}}^{*}\left(\frac{\partial}{\partial \mathrm{t}}\left(\rho_{\mathrm{t}}\right) \omega\right)$ 
Then (14) takes the form

$$
\int_{\mathrm{D}}\left[\phi_{\mathrm{t}}^{*}\left(\mathrm{~L}_{\mathrm{X}_{\mathrm{t}}}\left(\rho_{\mathrm{t}} \omega\right)+\frac{\partial}{\partial \mathrm{t}}\left(\rho_{\mathrm{t}}\right) \omega\right)\right]=0
$$

by change of variable formula

$$
\int_{\phi_{t}(D)}\left[L_{X_{t}}\left(\rho_{t} \omega\right)+\frac{\partial}{\partial t}\left(\rho_{t}\right) \omega\right]=0 ; \quad \forall D
$$

Since D is an arbitrary open set, this can be true only if the integrand is zero; that is

$$
\mathrm{L}_{\mathrm{X}}\left(\rho_{\mathrm{t}} \omega\right)+\frac{\partial}{\partial \mathrm{t}}\left(\rho_{\mathrm{t}}\right) \omega=0
$$

This is the equation of continuity in invariant form.Equation (17) can be written as

$$
\mathrm{L}_{\mathrm{t}} \omega_{\mathrm{t}}+\frac{\partial \omega_{\mathrm{t}}}{\partial \mathrm{t}}=0
$$

where $\omega_{t}:=\rho_{t} \omega$ is a 1-parameter family of $n$-forms on $M$.

(17) takes the following form

$$
\mathrm{L}_{\mathrm{X}}(\rho \omega)=0
$$

From properties of the Lie derivative this reduces to

$$
\left(L_{X} \rho\right) \omega+\rho L_{X} \omega=0
$$

Since $\rho$ is a constant function,the first term on the left-hand side of equation (20) is vanish, then equation (20) take the following form

$$
\mathrm{L}_{\mathrm{X}} \omega=0
$$

This is the geometric form of equation of continuity for incompressible fluid.According to (Cartan's formula) the Lie-derivative applied to volume form can may be written as

$$
L_{X} \omega=i_{x} d \omega+d i_{x} \omega=d i_{x} \omega
$$

Since $\mathrm{d} \omega=0$. Using (22), we find the alternative invariant formulation of continuity equation for incompressible fluid

$$
\operatorname{di}_{X_{t}} \omega=0
$$

These two formulations are equivalent.

Now, whenever $M=\mathbb{R}^{3}$. In fact

$$
\left.\begin{array}{c}
\mathrm{L}_{\mathrm{X}}\left(\rho_{\mathrm{t}} \omega\right)=\left(\mathrm{L}_{\mathrm{X}_{\mathrm{t}}} \rho_{\mathrm{t}}\right) \omega+\rho_{\mathrm{t}} \mathrm{L}_{\mathrm{t}} \omega=\mathrm{X}_{\mathrm{t}}\left(\rho_{\mathrm{t}}\right) \omega+\rho_{\mathrm{t}}\left(\operatorname{div} \mathrm{X}_{\mathrm{t}}\right) \omega \\
\mathrm{X}_{\mathrm{t}}\left(\rho_{\mathrm{t}}\right)=\sum \mathrm{v}^{\mathrm{i}}(\mathrm{x}, \mathrm{t}) \frac{\partial \rho}{\partial \mathrm{x}^{\mathrm{i}}}(16) \\
\operatorname{div} \mathrm{X}_{\mathrm{t}}=\frac{\partial \mathrm{v}^{\mathrm{i}}}{\partial \mathrm{x}^{\mathrm{i}}} \\
\frac{\partial}{\partial \mathrm{t}}\left(\rho_{\mathrm{t}}\right) \omega=\frac{\partial \rho}{\partial \mathrm{t}} \omega .
\end{array}\right\}
$$

substituting (24)into the continuity equation (17), we get the following

$$
\frac{\partial \rho}{\partial \mathrm{t}} \omega+\sum \mathrm{v}^{\mathrm{i}} \frac{\partial \rho}{\partial \mathrm{x}^{\mathrm{i}}} \omega+\rho \frac{\partial \mathrm{v}^{\mathrm{i}}}{\partial \mathrm{x}^{\mathrm{i}}} \omega=0
$$

Since $\omega=\mathrm{dx}^{1} \mathrm{dx}^{2} \mathrm{dx}^{3}$ (volume form), therefore

$$
\frac{\partial \rho}{\partial t}+\operatorname{div}(\rho v)=0
$$

Which is the usual equation of continuity. 


\subsection{Continuity Equation as Exterior Differential System (Verhulst, F., 2006)}

In this sub section, we use the Cartan's theory to show that it is possible to rewrite the continuity equation as an exterior differential system.

\section{A. Compressible (general) case:}

To reformulate the continuity equation as an exterior differential system(alternate geometric approach), we set.

$$
\left.\begin{array}{l}
\theta^{1}=d x^{1}-v^{1} d t \\
\theta^{2}=d x^{2}-v^{2} d t \\
\theta^{3}=d x^{3}-v^{3} d t
\end{array}\right\}
$$

We define the 3-form:

$$
\begin{aligned}
& \omega=\rho\left(d x^{1}-v^{1} d t\right) \wedge\left(d x^{2}-v^{2} d t\right) \wedge\left(d x^{3}-v^{3}\right) \\
& =\rho d x^{1} d x^{2} d x^{3}-\rho v^{1} d x^{1} d x^{2} d x^{3} d t+\rho v^{2} d x^{3} d t d x^{1}-\rho v^{3} d t d x^{1} d x^{2}
\end{aligned}
$$

By applying the exterior derivative of both sides in (28)

$$
\begin{gathered}
d \omega=d\left[\rho d x^{1} d x^{2} d x^{3}-\rho v^{1} d x^{1} d x^{2} d x^{3} d t+\rho v^{2} d x^{3} d t d x^{1}-\rho v^{3} d t d x^{1} d x^{2}\right] \\
\left.=d \rho \wedge d x^{1} d x^{2} d x^{3}-d\left(\rho v^{1}\right) \wedge d x^{2} d x^{3} d t+d\left(\rho v^{2}\right) \wedge d x^{3} d t d x^{1}-d\left(\rho v^{3}\right) \wedge d t d x^{1} d x^{2}\right] \\
=\left(\frac{\partial \rho}{\partial t} d t+\frac{\partial \rho}{\partial x^{1}} d x^{1}+\frac{\partial \rho}{\partial x^{2}} d x^{2}+\frac{\partial \rho}{\partial x^{3}} d x^{3}\right) d x^{1} d x^{2} d x^{3} \\
-\left(\frac{\partial \rho v^{1}}{\partial t} d t+\frac{\partial \rho v^{1}}{\partial x^{1}} d x^{1}+\frac{\partial \rho v^{1}}{\partial x^{2}} d x^{2}+\frac{\partial \rho v^{1}}{\partial x^{3}} d x^{3}\right) d x^{2} d x^{3} d t \\
+\left(\frac{\partial \rho v^{2}}{\partial t} d t+\frac{\partial \rho v^{2}}{\partial x^{1}} d x^{1}+\frac{\partial \rho v^{2}}{\partial x^{2}} d x^{2}+\frac{\partial \rho v^{2}}{\partial x^{3}} d x^{3}\right) d x^{3} d t d x^{1} \\
-\left(\frac{\partial \rho v^{3}}{\partial t} d t+\frac{\partial \rho v^{3}}{\partial x^{1}} d x^{1}+\frac{\partial \rho v^{3}}{\partial x^{2}} d x^{2}+\frac{\partial \rho v^{3}}{\partial x^{3}} d x^{3}\right) d t d x^{1} d x^{2}
\end{gathered}
$$

Using the properties of wedge product, this becomes

$$
\begin{gathered}
d \omega=\frac{\partial \rho}{\partial t} d t d x^{1} d x^{2} d x^{3}-\frac{\partial \rho v^{1}}{\partial x^{1}} d x^{1} d x^{2} d x^{3} d t+\frac{\partial \rho v^{2}}{\partial x^{2}} d x^{2} d x^{3} d t d-\frac{\partial \rho v^{3}}{\partial x^{3}} d x^{3} d t d x^{1} d x^{2} \\
=\left[\frac{\partial \rho}{\partial t}+\frac{\partial \rho v^{1}}{\partial x^{1}}+\frac{\partial \rho v^{2}}{\partial x^{2}}+\frac{\partial \rho v^{3}}{\partial x^{3}}\right] d t d x^{1} d x^{2} d x^{3}
\end{gathered}
$$

Therefore $\operatorname{div} \omega=0$ corresponds to

$$
\frac{\partial \rho}{\partial t}+\frac{\partial \rho v^{1}}{\partial x^{1}}+\frac{\partial \rho v^{2}}{\partial x^{2}}+\frac{\partial \rho v^{3}}{\partial x^{3}}=0
$$

Thus the continuity equation correspond to the closed form

$$
d \omega=0
$$

Suppose we express the flow in terms of initial conditions (or other parameters) by

so that the a' are the parameters and

$$
x=x\left(t, \alpha^{1}, \ldots, \alpha^{3}\right),
$$

$$
\frac{\partial x}{\partial t}=v,
$$

$$
\text { Thus } d x^{i}-v^{i} d t=\left(\frac{\partial x^{i}}{\partial t} d t+\sum \frac{\partial x^{i}}{\partial \alpha^{j}} d \alpha^{j}\right)-v^{i} d t=\sum \frac{\partial x^{i}}{\partial \alpha^{j}} d \alpha^{j}
$$

So that

$$
\omega=\rho \frac{\partial\left(x^{1}, x^{2}, x^{3}\right)}{\partial\left(\alpha^{1}, \alpha^{2}, \alpha^{3}\right)} d \alpha^{1} d \alpha^{2} d \alpha^{3}=K(t, \alpha) d \alpha^{1} d \alpha^{2} d \alpha^{3}
$$

Since $d \omega=0 \quad$ we deduce that 


$$
\frac{\partial k}{\partial t}=0
$$

Therefore

$$
\omega=A(\alpha) d \alpha^{1} d \alpha^{2} d \alpha^{3} .
$$

This means that the 3-form $\omega$ is an integral-invariant for the flow $\phi_{t}$, which represents property of conservation of mass Equation (32).We consequently have the following result. Given two 3-chains $C_{3}, C_{3}^{\prime} \in C_{3}(M)$ which are in $1-1$ correspondence such that corresponding points lie on the same trajectory of the flow $\left\{\phi_{t}\right\}$, then:

$$
\int_{C_{3}} \omega=\int_{C_{3}^{\prime}} \omega, \quad C_{3}^{\prime}=\left(\phi_{t}\right)_{*} C_{3}
$$

If now $C_{3}=C_{3}^{(0)}$ at $t_{0}=0$, then, by (2.37),

$$
\omega=\left.\int_{C_{3}^{\prime}} \omega\right|_{t=t_{0}}=\int_{C_{3}} \rho d x^{1} d x^{2} d x^{3},
$$

And following up $C_{3}^{(0)}=C_{3}^{(1)}$ at time $t_{1}$, we have:

$$
\int_{C_{3}^{(0)}} \rho d x^{1} d x^{2} d x^{3}=\int_{C_{3}^{(1)}} \rho d x^{1} d x^{2} d x^{3}
$$

Which expresses that mass is preserved in the flow $\left\{\phi_{t}\right\}$, another form of the conservation of mass.

\section{B. Inviscid, incompressible, and irrotational (potential flow) case:}

A potential flow describes what the flow would be like if it were inviscid, incompressible, and irrotational. which in vector calculus is described by Laplace equation $\nabla^{2} \varphi=$.Reformulating the problem in a differential geometry terms we consider the scalar function $\varphi$ which is a 0 - form or a 2 - form, following contact variables

$$
p=\frac{\partial \varphi}{\partial x}, q=\frac{\partial \varphi}{\partial y}, r=
$$

Let $\mathrm{M}$ be a manifold with variables $(x, y, z, \varphi, p, q, r)$, on $\mathrm{M}$ we define the contact form

$$
\alpha=d \varphi-p d x-q d y-r d z
$$

And the 2-form

$$
\alpha=p d y d z+q d z d x+r d x d y,
$$

By taking the exterior derivative of (44) and using the anti-symmetric property of wedge product we obtain

$$
\begin{gathered}
d \sigma=d[p d y d z+q d z d x+r d z d y]=d p \wedge d y d z+d q \wedge d z d x+d r \wedge d x d y \\
=\left(\frac{\partial p}{\partial x} d x+\frac{\partial p}{\partial y} d y+\frac{\partial p}{\partial z} d z\right) d y d z+\left(\frac{\partial q}{\partial x} d x+\frac{\partial q}{\partial y} d y+\frac{\partial q}{\partial z} d z\right) d z d x \\
+\left(\frac{\partial r}{\partial x} d x+\frac{\partial r}{\partial y} d y+\frac{\partial r}{\partial z} d z\right) d x d y
\end{gathered}
$$

By using the anti-symmetric property of wedge product, this becomes

$$
d \sigma=\frac{\partial p}{\partial x} d x d y d z+\frac{\partial q}{\partial y} d y d z d y+\frac{\partial r}{\partial z} d z d x d y=\left[\frac{\partial^{2} \varphi}{\partial x^{2}}+\frac{\partial^{2} \varphi}{\partial y^{2}}+\frac{\partial^{2} \varphi}{\partial z^{2}}\right] d x d y d z
$$

Note that $d \sigma=0$. Then Laplace equation is equivalent to the relation

$$
d \sigma=0
$$

This is the coordinate-free version.

\section{Equations of Motion}

5.1 Momentum Equation on Manifolds (Datta, A., \& Majumdar, A., 1980)

Navier-Stokes equation is the most general equation for description of fluid phenomena, which as special case comprises Euler's equation of motion.Let $\mathrm{M}$ be a differentiable n-manifold, $\theta$ be a differential 1-form on $\mathrm{M}$, co be a volume-element differential form on Mand $\mathrm{D}$ be a domain in $\mathrm{M}$.

Consider a fluid moving in a domain $D \subset M$. For any continuum there are two types of forces acting on a piece of material. 
1) First there are external or body forces:

whereF is a vector field representing the volume forces on a domain $\mathrm{D}$, the $\theta$-component of the volume forces acting on the domain $\mathrm{D}$ are

$$
\text { Force }_{\text {vol }}=\int_{D} \rho^{\theta}(F) \omega,
$$

2) Second there are stress forces (discipline of continuum mechanics can also encounter forces that come from the region surrounding a bit of fluid, expressed by the stress tensor):

Phrasing this stress force a differential geometric language.

Let $\mathrm{T}$ be a tensor field on $\mathrm{M}$ representing this stress. At $x \in M, \mathrm{~T}$ is a skew-symmetric tensor field on $\mathrm{M}$ define in the following form:

$$
\theta^{n-1}\left(X_{1}, \ldots, X_{n-1}\right)=\theta\left(T\left(X_{1}, \ldots, X_{n-1}\right)\right) ; X_{i} \in T_{x}
$$

Then we define the stress tensor of the fluid inside $D \subset M$ in terms of the multilinear map:

$$
T=T\left(X_{1}, X_{2}, \ldots, X_{n-1}\right): T_{x}(M) \times \ldots \times T_{x}(M) \rightarrow T_{x}(M),
$$

For $x \in M, X_{1}, X_{2}, \ldots, X_{n-1} \in T_{x}(M)$

$\left(T\left(X_{1}, \ldots, X_{n-1}\right)\right)$ is an $(n-1)$ covector on M. Then $\theta(T)$ defines for each $\theta$ an $(n-1)$-differential form on M. If D is a domain in $\mathrm{M}$ with boundary $\partial D$, then the total $\theta$-component of the stress force is

Applying Stokes' theorem, to $\int_{\partial D} \theta(T)$ we get

$$
\text { Force }_{\text {str }}=\int_{\partial D} \theta(T) \text {, }
$$

$$
\text { Force }_{\text {str }}=\int_{\partial D} \theta(T)=\int_{D} d \theta(T)
$$

From (11) and (15) the 8-component of the force acting on the domain D at fixed time is

$$
\text { Force }_{\text {tot }}=\int_{\partial D}\left[\rho \theta(F) \omega+d \theta(T)=\int_{\phi_{t}(D)}\left[\rho_{t} \theta\left(F_{t}\right) \omega+d\left(\theta\left(T\left(z_{t}\right)\left(i_{z} \omega\right)\right)\right)\right]\right.
$$

Where $\phi_{t}(D)$ is a volume preserving fluid flow with evolution operator $\phi_{t}$.

Suppose a group of particles making up the fluid start out at $\mathrm{t}=0$ to occupy the domain $\mathrm{D}$. At time $\mathrm{t}$, they will be in domain $\phi_{t}(D)$, their $\theta$ - component of total momentum will be

by the change of variables theorem, this equals

$$
\text { Momentum }_{\text {tot }}=\int_{\phi_{t}(D)} \theta\left(X_{t}\right) \rho_{t} \omega,
$$

$$
\text { Momentum }_{\text {tot }}=\int_{D} \phi_{t}^{*}\left(\theta\left(X_{t}\right) \rho_{t} \omega\right) .
$$

The rate of change of momentum

$$
\begin{gathered}
\frac{\partial}{\partial t} \int_{D} \phi_{t}^{*}\left(\theta\left(X_{t}\right) \rho_{t} \omega\right)=\int_{D} \frac{\partial}{\partial t}\left[\phi_{t}^{*}\left(\theta\left(X_{t}\right) \rho_{t} \omega\right)\right] \\
=\int_{D} \phi_{t}^{*}\left(L_{X_{t}}\left(\theta\left(X_{t}\right) \rho_{t} \omega\right)\right)+\int_{D} \phi_{t}^{*}\left(\theta\left(\frac{\partial X_{t}}{\partial t}\right) \rho_{t} \omega+\theta\left(X_{t}\right) \frac{\partial \rho_{t}}{\partial t} \omega\right)
\end{gathered}
$$

By the change of variables theorem, we get

$$
\frac{\partial}{\partial t} \int_{D} \phi_{t}^{*}\left(\theta\left(X_{t}\right) \rho_{t} \omega\right)=\int_{\phi_{t}(D)}\left[L_{X_{t}}\left(\theta\left(X_{t}\right) \rho_{t} \omega\right)+\theta\left(\frac{\partial X_{t}}{\partial t}\right) \rho_{t} \omega+\theta\left(X_{t}\right) \frac{\partial \rho_{t}}{\partial t} \omega\right]
$$

By applying Newton's law of motion (principle of balance of momentum): the rate of momentum of a portion of the fluid equals the total force applied to it. By equating (16) to the expression (20) for the force acting in this region, we get

$$
\begin{gathered}
\int_{\phi_{t}(D)}\left[\rho_{t} \theta\left(F_{t}\right) \omega+d\left(\theta\left(T\left(z_{t}\right)\left(i_{z} \omega\right)\right)\right)\right] \\
=\int_{\phi_{t}(D)}\left[L_{X_{t}}\left(\theta\left(X_{t}\right) \rho_{t} \omega\right)+\theta\left(\frac{\partial X_{t}}{\partial t}\right) \rho_{t} \omega+\theta\left(X_{t}\right) \frac{\partial \rho_{t}}{\partial t} \omega\right]
\end{gathered}
$$

Since this relation is to hold for all domainsD, we have 


$$
\rho_{t} \theta\left(F_{t}\right) \omega+d \theta\left(T_{t}\right)=L_{X_{t}}\left(\theta\left(X_{t}\right) \rho_{t} \omega\right)+\theta\left(\frac{\partial X_{t}}{\partial t}\right) \rho_{t} \omega+\theta\left(X_{t}\right) \frac{\partial \rho_{t}}{\partial t} \omega
$$

This is the geometric version of momentum equation of fluid motion, which is coordinate-free.

If $\mathrm{M}=\mathbb{R}^{3}$, with coordinates $x_{i}, i=1,2,3$.

$$
\begin{gathered}
X_{i}=v_{i}(x, t) \frac{\partial}{\partial x_{i}}, \quad \rho_{t}=\rho(x, t), \\
\omega=d x_{1} \wedge d x_{2} \wedge d x_{3}, \quad \theta=d x_{k}, \\
F=F^{k} \frac{\partial}{\partial x_{i}}, \quad \theta(\mathrm{F})=d x_{k}(F)=d x_{k}\left(F^{i} \frac{\partial}{\partial x_{i}}\right)=F^{k}
\end{gathered}
$$

We consider the bilinear map:

$$
\begin{gathered}
T_{x}(M) \times T_{x}(M) \rightarrow T_{x}(M) \\
(X, Y) \rightarrow T(X, Y)(x)=\sum X^{i} Y^{j} T\left(\frac{\partial}{\partial x^{i}}, \frac{\partial}{\partial x^{j}}\right)
\end{gathered}
$$

Defined by

$$
T_{x}(X(x), Y(x))=T(X, Y)(x)
$$

Where

$$
\begin{gathered}
X=\sum X^{i} \frac{\partial}{\partial x^{i}}, \quad Y=\sum Y^{j} \frac{\partial}{\partial x^{j}}, \quad X, Y \in \mathfrak{X}(M) \\
T\left(\frac{\partial}{\partial x^{i}}, \frac{\partial}{\partial x^{j}}\right)=T_{i j k} \frac{\partial}{\partial x^{k}},(2.3 .15)
\end{gathered}
$$

Then

$$
\begin{gathered}
T(X, Y)(x)=X^{i} Y^{j} T\left(\frac{\partial}{\partial x^{i}}, \frac{\partial}{\partial x^{j}}\right)(x)=X^{i}(x) Y^{j}(x) T_{i j k}(x) \frac{\partial}{\partial x^{k}}, \\
\text { And } T_{i j k}=\frac{1}{2} \in_{i j_{1}} T_{1 k},
\end{gathered}
$$

Where $\epsilon_{i j_{1}}$ is the 3-index, skew-symmetric tensor (with $\epsilon_{123}=1$ ). Equations (63) and (64) correspond to the conventional continuous approach where:

$$
T_{k}(d S)=T_{i j_{1}} d S^{i j},
$$

Now

$$
\begin{gathered}
d(\theta(T))+\frac{1}{2} \frac{\partial T_{i j k}}{\partial x_{h}} d x_{h} \wedge d x_{j}=\frac{1}{2}\left(\epsilon_{h i j} \frac{\partial T_{i j k}}{\partial x_{h}}\right) \omega \\
=\frac{1}{2} \epsilon_{h i j} \in_{i j 1} \frac{\partial T_{i k}}{\partial x_{h}} \omega=\delta_{h 1} \frac{\partial T_{i k}}{\partial x_{h}} \omega=\frac{\partial T_{i k}}{\partial x_{h}}, \\
\text { And } X_{t}(\omega)=\frac{\partial v_{i}}{\partial x_{i}} \omega
\end{gathered}
$$

Hence (66) becomes

$$
\rho F_{k}+3(\omega)=\frac{\partial T_{i k}}{\partial x_{i}}=v_{i} \frac{\partial}{\partial x_{i}}\left(v_{k} \rho\right)+v_{k} \rho \frac{\partial v_{i}}{\partial x_{i}}+\frac{\partial v_{k}}{\partial t} \rho+v_{k} \frac{\partial \rho}{\partial t},
$$

Which is the usual momentum equation of motion.

5.2 Euler Equation on Riemannian Manifold (Gilkey, P. B., 1975; Yamabe, H., 1960)

In this section we express the Euler's equation in the language of differential one-forms on Riemannian manifold. The formulation is obtained in the absence of body forces. 
Consider an n-dimensional manifold $\mathrm{M}$ with a Riemannian metric $\mathrm{g}$. By identifying the differential forms with their dual vector fields, we rewrite Euler equation in terms of differential 1-forms. Let $\alpha \in \Omega^{1}(M)$ be the one form associated to X ( $\alpha$ is the one-form dual to $\mathrm{X}$ ), describe the velocity of an ideal fluid. We seek an invariant meaning for the sum of the last three terms on the left-hand side of equation (26). For fixed i this expression equals

$$
v_{j} \frac{\partial v_{i}}{\partial x^{j}}=v_{j} \frac{\partial v_{i}}{\partial x^{j}}+v_{j} \frac{\partial v_{j}}{\partial x^{i}}-v_{j} \frac{\partial v_{j}}{\partial x^{i}}=L_{x} \alpha-\frac{1}{2} d(\alpha(x))(27) \mathrm{Also} \frac{\partial p}{\partial x_{i}}=d p
$$

So substituting (67) and (68) into the Euler equations (65), we get the following exterior differential system

$$
\frac{\partial \alpha}{\partial t}+L_{x} \alpha-\frac{1}{2} d(\alpha(x))+\frac{1}{\rho} d P=0
$$

This is the Euler equation in terms of differential 1-forms. According to (Cartan's magic formula) the Lie-derivative applied to one-form a may be written as

$$
\begin{aligned}
L_{x} \alpha & =i_{X} d \alpha+d i_{X} \alpha \\
& =i_{X} d \alpha+d(\alpha(x)) \\
& =i_{X} \varpi+d(\alpha(x))
\end{aligned}
$$

Since $d \alpha=\varpi$. So substituting (70) into the Euler equation (69), we get the following alternative form

$$
\frac{\partial \alpha}{\partial t}+i_{X} \varpi+\frac{1}{2} d(\alpha(x))+\frac{1}{\rho} d P=0
$$

On the basis of the Euler equation (69)and exterior calculus, we derive in the following a set of invariant fluid equations.

\section{A. Bernoulli equation.}

For a steady (time-independent) flow of a perfect (an inviscid and incompressible) fluid, Euler equation (69) becomes

By using Cartan Magic formula

$$
L_{x} \alpha=-d\left(\frac{1}{\rho} P-\frac{1}{2} \alpha(x)\right)
$$

$$
\begin{aligned}
L_{x} \alpha & =i_{X} d \alpha+d i_{X} \alpha \\
& =i_{X} d \alpha+d(\alpha(x))
\end{aligned}
$$

Substituting (73) into (72) we get

$$
\begin{aligned}
& i_{x} d \alpha=-d\left(\frac{1}{\rho} P-\frac{1}{2} \alpha(X)\right) \\
& \text { Where } \quad f=\frac{1}{\rho} P-\frac{1}{2} \alpha(X)
\end{aligned}
$$

From (74) we have

$$
i_{x} d\left(\frac{1}{\rho} P+\frac{1}{2} \alpha(X)\right)=0
$$

Since $i_{x} i_{x}=0$, so that the quantity $\frac{1}{\rho} P+\frac{1}{2} \alpha(X)$ is constant along each streamline (integral curves of $\mathrm{X}$ ) of the fluid flow. This is the Bernoulli's principle. The function $\mathrm{f}$ is known as the Bernoulli function of $\mathrm{X}$.

Also

meaning that $\mathrm{d} \alpha$ is preserved by $\mathrm{X}$.

$$
d\left(i_{x} d \alpha\right)=L_{x} d \alpha=d(-d f)=0
$$

$$
\text { Since } i_{x} d \alpha-d f \text {, we get } L_{x} f=-i_{X} i_{X} d \alpha=0
$$

Thus $\mathrm{f}$ is invariant under the flow of $\mathrm{X}$. We will call Euler vector fields to the solutions to the equations of an ideal steady incompressible fluid on a manifold.

If the fluid is irrotational $(d \alpha=0)$ then Poincare lemma tells us that

$$
\alpha=d \varphi
$$

Where $\varphi \in \mathcal{F}_{U}$ is a velocity potential, $U \subset M$. Substituting (79) into (71) we obtain 


$$
\frac{\partial d \varphi}{\partial t}+\frac{1}{2} d(\alpha(X))=-\frac{1}{\rho} d P
$$

Therefore,

$$
d\left[\frac{\partial d \varphi}{\partial t}+\frac{1}{2}(\alpha(X))+\frac{1}{\rho} P\right]=0
$$

Thus we have the Unsteady Bernoulli Equation form from the geometric point of view

$$
\begin{gathered}
\frac{\partial \varphi}{\partial t}+\frac{1}{2}(\alpha(X))+\frac{1}{\rho} P+c, \\
\text { on } U \subset M \text {, where } c \in \mathcal{F}_{U} \text { satisfied } d c=0
\end{gathered}
$$

\section{B. Vorticity Equation and Conservation Properties}

On the basis of the Euler equation (69) we show conservation of vorticity

By taking the exterior derivative of (69),

$$
\varpi=d \alpha \in \Omega^{2}(M)
$$

$$
d\left[\frac{\partial \alpha}{\partial t}+L_{x} \alpha-\frac{1}{2} d(\alpha(X))+\frac{1}{\rho} d P\right]=0
$$

As the Lie-derivative and the exterior derivative commute, we get the following

$$
\frac{\partial d \alpha}{\partial t}+L_{x} d \alpha-\frac{1}{2} d d(\alpha(X))+d\left(\frac{1}{\rho}\right) d d P=0
$$

For the case of incompressible flows

$$
\frac{\partial \varpi}{\partial t}+\mathrm{L}_{x} \varpi=0
$$

This equation is called vorticity equation From (85) it follows that

$$
: \frac{\partial}{\partial t}\left(\phi_{t}^{*} \varpi_{t}\right)=0
$$

So

$$
\phi_{t}^{*} \varpi_{t}=\varpi_{0}
$$

Showing that vorticity moves with the fluid.This is, via stokes theorem another way of phrasing Kelvin's theorem.

5.2.1 Kelvin Circulation Theorem. (Marsden, J., \& Weinstein, A., 1983)

Let $\mathrm{M}$ be a manifold and $l \subset M$ a smooth closed loop that is, a compact one-manifold. Let $X_{t}$ solve the Euler equations on $\mathrm{M}$ for ideal isotropic compressible or homogeneous incompressible flow and $l(t)$ be the image of $l$ at time $\mathrm{t}$ when each particlemoves under the flow $\phi_{t}$ of $X_{t}$; that is, $l(t)=\phi_{t}(l)$. Then the circulation is constant in time; that is,

$$
\frac{d}{d t} \int_{l(t)} \alpha=0
$$

Proof. Let $\phi_{t}$ be the flow of $X_{t}$. Then $(t)=\phi_{t}(l)$, and by change of variables theorem, (89) becomes

$$
\frac{d}{d t} \int_{\phi_{t}(l)} \alpha=\frac{d}{d t} \int_{l} \phi_{t}^{*} \alpha=\int_{l}\left[\phi_{t}^{*}\left(L_{X} \alpha\right)+\phi_{t}^{*}\left(\frac{\partial \alpha}{\partial t}\right)\right] .
$$

However, $L_{X} \alpha+\partial \alpha / \partial t$ is exact from the equations of motion and the integral of an exact form over a closed loop is zero. We now use Stokes theorem, which will bring in the vorticity. If $\Sigma$ is a surface (a two-dimensional submanifold of M) whose boundary is a closed contour $\mathrm{C}$, then Stokes theorem yields

$$
\Gamma_{C}=\int_{C} \alpha=\int_{\Sigma} d \alpha=\int_{\Sigma} \varpi
$$

Thus, as a corollary of the circulation theorem, we can conclude:

\subsubsection{Helmholtz Theorem.}

The flux of vorticity across a surface moving with the fluid is constant in time 


\section{Classification of Fluids According to Diffeomorophisms (Kiehn, R., 2002)}

We will now sketch a conceptual frame work for some of the geometric structure of fluids.

\subsection{Compressible Fluid}

Let $\mathrm{M}$ and $\mathrm{N}$ be differentiable manifolds of the same dimension. Let $\mathrm{L}$ denote an interval of positive real numbers. Let $\Gamma_{f l}(M \times L, N)$ denote the set of all maps:

Such that $\phi \phi$ is smooth. For each t EL, the map

$$
\phi: M \times L \rightarrow N \quad:(x, t) \mapsto \phi(x, t),
$$

$$
\phi_{t}: M \rightarrow N \quad: x \mapsto \phi(x, t)
$$

is a diffeomorphism.

6.1.1 Definition

$\Gamma_{f l}(M \times L, N)$ is called the space of smooth fluid flows in $\mathrm{N}$ parameterized by M. geometrically, $t \mapsto \phi_{t}$ can be considered as a one parameter deformation in the space of diffeomorphisms, we can write:

$$
\phi_{t}=\phi^{\circ} c_{t}
$$

Where $t \mapsto c_{t} \in \operatorname{Diff}(\mathrm{N})$ is a curve in the group of all diffeomorphisms of $\mathrm{N}$. recall the ideas of the jet bundle calculus. We do this in a form that accords with intuition surrounding what is called the Eulerian picture in fluid mechanics.

Let $\mathrm{M}$ and $\mathrm{N}$ be finite dimensional, infinitely-differentiable manifold. Let $\phi_{f l}(M \times L, N)$ be as above, introduce the following equivalence relation on $M \times L \times \Gamma_{f l}(M \times L, N)$ :

$$
\left.\begin{array}{rl}
(x, t, \phi) & =\left(x^{\prime}, t^{\prime}, \phi^{\prime}\right) \text { iff } \\
x & =x^{\prime} \\
t & =t^{\prime} \\
\phi(x, t) & =\phi^{\prime}(x, t)
\end{array}\right\}
$$

The curves $s \rightarrow \phi(x, s)$ and $s \rightarrow \phi^{\prime}(x, s)$ have the same tangent vector at $s=t$.

\subsubsection{Definition}

Let $E(M, N)$ be the quotient set of $M \times L \times \Gamma_{f l}(M \times L, N)$ with respect to this equivalence relation. $E(M, N)$ is called the Eulerian velocity bundle for motion of fluids in $\mathrm{N}$ parameterized by $\mathrm{M}$. It is readily seen to be a manifold.

\subsubsection{Definition}

Given $(\mathrm{x}, \mathrm{t}, \phi) \in \mathrm{M} \times \mathrm{L} \times \Gamma_{\mathrm{fl}}(\mathrm{M} \times \mathrm{L}, \mathrm{N})$, the equivalence class to which the pair $(\mathrm{x}, \phi)$ belongs is denoted as $\mathrm{v}_{(\phi)(\mathrm{x}, \mathrm{t})} \cdot \operatorname{as}(\mathrm{x}, \mathrm{t})$ varies, we obtain a map.

Called the Eulerian velocity field associated with the fluid flow $\phi$. Consider the target map

$$
\mathrm{v}_{\phi}: \mathrm{M} \times \mathrm{L} \rightarrow \mathrm{E}(\mathrm{M}, \mathrm{N}),
$$

Called the Eulerian velocity field associated with the fluid flow $\phi$. Consider the target map:

$$
\begin{gathered}
\mathrm{M} \times \mathrm{L} \times \Gamma_{\mathrm{fl}}(\mathrm{M} \times \mathrm{L}, \mathrm{N}) \rightarrow \mathrm{N} \times \mathrm{L} \\
:(\mathrm{x}, \mathrm{t}, \phi) \mapsto \phi(\mathrm{x}, \mathrm{t})
\end{gathered}
$$

This map is constant on the equivalence relation (6), hence defines a quotient map that we will label as follows:

$$
\pi_{\operatorname{tar}} \mathrm{E}(\mathrm{M}, \mathrm{N}) \rightarrow \mathrm{N} \times \mathrm{L}
$$

The fibers of $\pi_{\text {tar }}$ are tangent vectors to $N$, hence $\pi_{\text {tar }}$ defines $E(M, N)$ as a vector bundle over $N \times L$ where TN is the tangent bundle to $\mathrm{N}$.

\subsection{Incompressible Fluids}

Let us define the geometry of an incompressible fluid. Let $\eta$ and $\omega$ denote fixed volume-element differential forms on $\mathrm{N}$ and $\mathrm{M}$, respectively.

Let $\Gamma_{\text {infl }}(M \times L, N)$ denote the set of all maps

$$
\begin{gathered}
\phi: M \times L \rightarrow N \\
:(x, t) \longmapsto \phi(x, t),
\end{gathered}
$$

Such that $\phi \in \Gamma_{\mathrm{fl}}(\mathrm{M} \times \mathrm{L}, \mathrm{N})$, for each $t \in L$, the map:

$$
\phi_{\mathrm{t}}: \mathrm{M} \rightarrow \mathrm{N} \quad: \mathrm{x} \longmapsto \phi(\mathrm{x}, \mathrm{t}),
$$


Is a diffeomorphism, and satisfies: $\phi_{\mathrm{t}}^{*}(\eta)=\omega$.

\section{References}

Arkani-Hamed, N., Cachazo, F., \& Kaplan, J. (2010). What is the simplest quantum field theory? Journal of High Energy Physics, 9, 1-92.

Bajura, R., \& Jones, E. (1976) Flow distribution manifolds. Journal of Fluids engineering, 98(4), 654-665.

Beig, R. Differential Geometry And Lie Groups Application. Initiativkolleg Der Universitat Wien.

Datta, A., \& Majumdar, A. (1980). Flow distribution in parallel and reverse flow manifolds. International Journal of Heat and Fluid Flow, 2(4), 253-262.

Erbar, M. (2010). The heat equation on manifolds as a gradient flow in the Wasserstein space. In Annales de l'institut Henri Poincaré $(B)$.

Gilkey, P. B. (1975). The boundary integrand in the formula for the signature and Euler characteristic of a Riemannian manifold with boundary. Advances in Mathematics, 15(3), 334-360.

Haller, G. (2001). Distinguished material surfaces and coherent structures in three-dimensional fluid flows. Physica D: Nonlinear Phenomena, 149(4), 248-277.

Kiehn, R. (2002). Holonomic and Anholonomic Constraints and Coordinates. Frobenius Integrability and Torsion of Various Types. Technical Report, University of Houston.

Kobayashi, S., \& Nomizu, K. (1963). Foundations of differential geometry.

Kolár, I., Slovák, J., \& P. W. (1999). Michor, Natural operations in differential geometry.

Lang, S. (1999). Fundamentals of Differential Geometry. Graduate Texts in Mathematics., Springer-Verlag, 191.

Marsden, J., \& Weinstein, A. (1983). Coadjoint orbits, vortices, and Clebsch variables for incompressible fluids. Physica D: Nonlinear Phenomena, 7(1), 305-323.

O'neill, B. (1983). Semi-Riemannian Geometry With Applications to Relativity, 103. Academic press.

Verhulst, F. (2006). Nonlinear differential equations and dynamical systems. Springer Science \& Business Media.

Warner, F. W. (2013). Foundations of differentiable manifolds and Lie groups. Springer Science and Business Media, 94.

Yamabe, H. (1960). On a deformation of Riemannian structures on compact manifolds.

\section{Copyrights}

Copyright for this article is retained by the author(s), with first publication rights granted to the journal.

This is an open-access article distributed under the terms and conditions of the Creative Commons Attribution license (http://creativecommons.org/licenses/by/4.0/). 